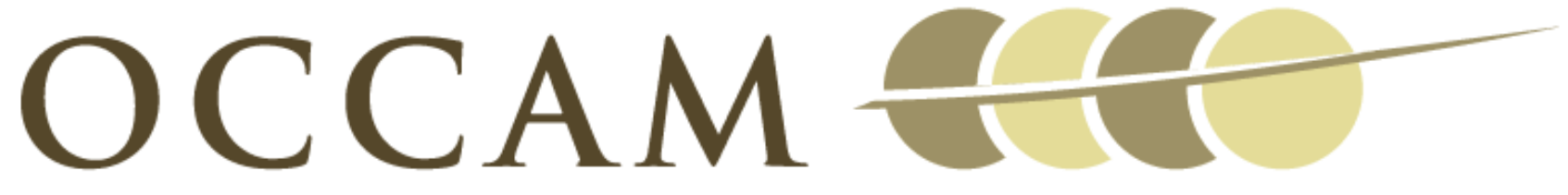

OXFORD CENTRE FOR COLLABORATIVE APPLIED MATHEMATICS

Report Number 12/30

A theoretical investigation of the effect of proliferation and adhesion on monoclonal conversion in the colonic crypt

by

Gary R. Mirams, Alexander G. Fletcher, Philip K. Maini, Helen M. Byrne

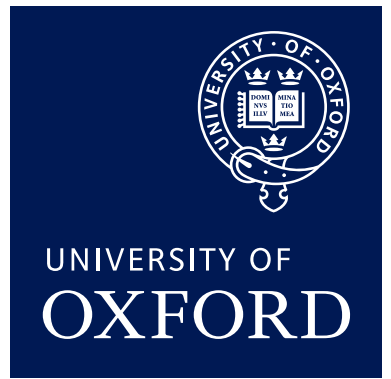

Oxford Centre for Collaborative Applied Mathematics Mathematical Institute 24 - 29 St Giles'

Oxford

OX1 3LB

England 



\title{
A theoretical investigation of the effect of proliferation and adhesion on monoclonal conversion in the colonic crypt
}

\author{
Gary R. Mirams ${ }^{\mathrm{a}, 1, *}$, Alexander G. Fletcher ${ }^{\mathrm{b}, \mathrm{c}, 1}$, Philip K. Maini ${ }^{\mathrm{b}, \mathrm{c}}$, Helen M. Byrne ${ }^{\mathrm{a}, \mathrm{d}}$ \\ ${ }^{a}$ Computational Biology, Department of Computer Science, \\ University of Oxford, Parks Road, Oxford, OX1 3QD, UK \\ ${ }^{b}$ Centre for Mathematical Biology, Mathematical Institute, \\ University of Oxford, St. Giles', Oxford, OX1 3LB, UK \\ ${ }^{c}$ Oxford Centre for Integrative Systems Biology, Department of Biochemistry, \\ University of Oxford, South Parks Road, Oxford OX1 3QU, UK \\ ${ }^{d}$ Oxford Centre for Collaborative Applied Mathematics, Mathematical Institute, \\ University of Oxford, St. Giles', Oxford, OX1 3LB, UK
}

\begin{abstract}
Colorectal cancers are initiated by the accumulation of mutations in the colonic epithelium. Using a spatially structured cell-based model of a colonic crypt, we investigate the likelihood that the progeny of a mutated cell will dominate, or be sloughed out of, a crypt. Our approach is to perform multiple simulations, varying the spatial location of the initial mutation, and its proliferative and adhesive properties, to obtain statistical distributions for the probability of domination. Our simulations lead us to make a number of predictions. The process of monoclonal conversion always occurs, and does not require that the cell which initially gave rise to the population remains in the crypt. Mutations occurring more than one to two cells from the base of the crypt are unlikely to become the dominant clone. The probability of a mutant clone persisting in the crypt is sensitive to dysregulation of adhesion, and comparison with a one-dimensional model suggests that this is caused by competition directly at the base of the crypt. We also predict that increases in the extent of the spatial domain in which the mutant cells proliferate cause counter-intuitive non-linear changes to the probability of its fixation, due to effects that cannot be captured in simpler models.
\end{abstract}

Keywords: mathematical model, colorectal crypt, stem cell, mutation, clonal expansion

\section{Introduction}

2 The intestinal epithelium is one of the most rapidly renewing mammalian tissues, with complete turnover 3 occurring every 2-3 days in mice and 3-5 days in humans (Okamoto and Watanabe, 2004; Ross et al., 4 2003). About $2 \times 10^{7}$ crypts of Lieberkühn form the epithelial layer of the human large intestine (Potten 5 et al., 2003). Topologically, crypts are 'test tube' or 'flask'-shaped invaginations in the epithelium of the 6 colon, providing a huge surface area for the absorption of water. Cells towards the base of the crypts

\footnotetext{
* Corresponding author

Email address: gary.mirams@cs.ox.ac.uk (Gary R. Mirams)

${ }^{1}$ Joint first authors 

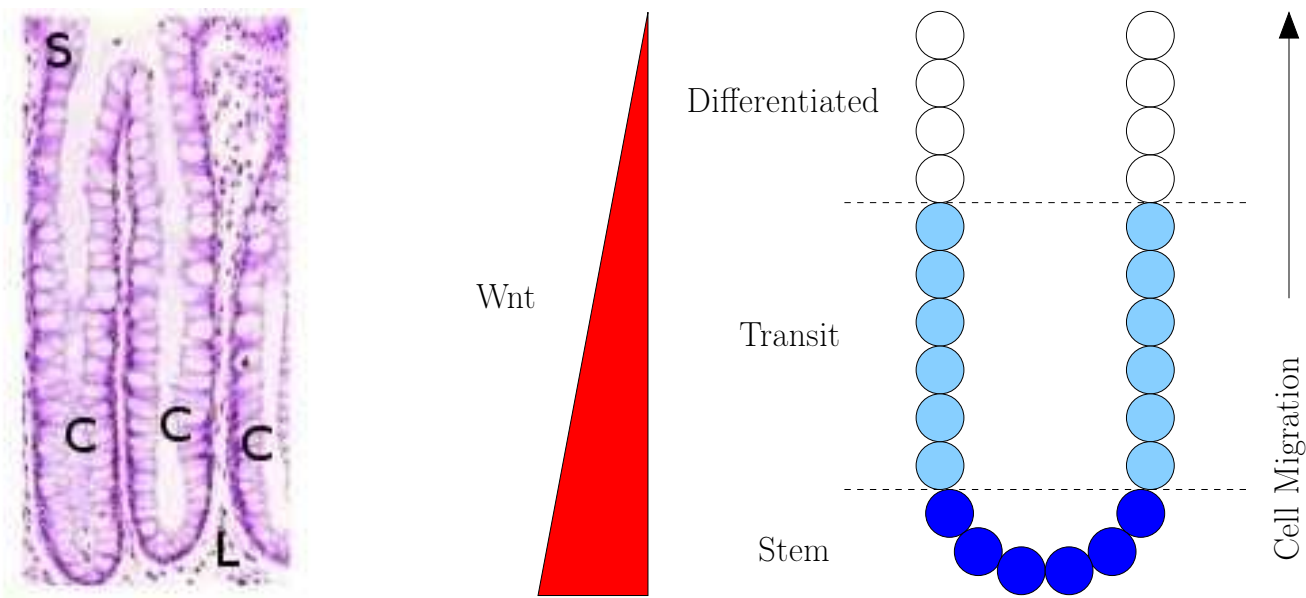

Figure 1: Left: Hematoxylin and eosin stained section through normal healthy human colonic mucosae, showing the testtube like structure of each crypt. Labels show surface epithelium (S), crypts (C) and the lamina propria separating them (L). Image reproduced from Shih et al. (2001), copyright (2001) National Academy of Sciences, USA. Right: Diagram indicating the spatial location of the stem, transit amplifying and differentiated cell compartments within the crypt. A Wnt gradient up the crypt is hypothesized to regulate cell proliferation.

7 proliferate. As cells ascend the crypt walls they cease proliferation and differentiate into specialised 8 absorptive or secretory cells. At the crypt orifice, differentiated cells undergo apoptosis and are shed into

\footnotetext{
${ }^{2}$ See http://www.stanford.edu/ rnusse/pathways/targets.html for an up-to-date list of target genes.
} 
migration of crypt cells, and to cause an increase in levels of proliferation (Sansom et al., 2004). This suggests that Wnt is a major controlling factor of cellular crypt dynamics. In support of this, mutations in key components of the Wnt pathway of epithelial crypt cells contribute to the initiation of over $90 \%$ of colorectal cancers, including most hereditary cases (Powell et al., 1992; Morin et al., 1997; Sparks et al., 1998; Ilyas, 2005; Barker et al., 2008). In this article we will simulate the mutation of single cells in the crypt, varying their proliferative and adhesive properties, along with the location of the mutation, in order to establish the probability that such mutants will dominate a colonic crypt.

As a crypt is a complex, highly-regulated system, a theoretical approach is useful for gaining mechanistic insights into dynamics that cannot be readily measured. Mathematical modelling has been used to investigate aspects of colorectal cancer for over half a century. But only over the last 10-20 years has the quality of experimental data advanced sufficiently to warrant the development of detailed models of the cell population dynamics within the crypt. Efforts include deterministic compartmental ordinary differential equation (ODE) models such as those by Johnston et al. (2007) and Boman et al. (2008), stochastic models such as those by Loeffler et al. (1993) and Komarova and Wang (2004), and more recent spatial models such as those by Meineke et al. (2001), van Leeuwen et al. (2009), Osborne et al. (2010) and Fletcher et al. (2012). Other models have considered the sub-cellular behaviour of normal and mutant crypt cells (van Leeuwen et al., 2007; Mirams et al., 2010), or some of the later steps in colorectal carcinogenesis (Drasdo and Loeffler, 2001; Edwards and Chapman, 2007). In another recent theoretical study, Buske et al. (2011) developed a spatial model of the intestinal crypt, which incorporated the effect of Wnt and Notch signalling on cell proliferative behaviour as well as an explicit representation of the basement membrane that provides mechanical support to the crypt.

An explanation of the role of Wnt and Delta-Notch signalling in stem cell control and cancer initiation can be found in reviews by, among others, Polakis (2007); Humphries and Wright (2008); van der Flier and Clevers (2009); Andersson et al. (2011). A detailed mathematical description of the pathways is not the focus of our study, as prediction of the phenotypic changes caused by mutations at the molecular level remains a very difficult task. Instead, we examine how alterations to the cellular-level properties, which mutations in the Wnt pathway may cause, affect the likelihood that a clone becomes dominant in the crypt.

The modular nature of the implementation of the van Leeuwen et al. (2009) crypt model enables us easily to use the Chaste platform (Cancer, Heart And Soft Tissue Environment, Pitt-Francis et al. (2009)) to perform large numbers of in silico experiments. Having studied the dynamics of a normal crypt, a next step is to consider the pathological behaviour that arises when crypt cells accumulate genetic mutations. In this paper we will investigate crypt homeostasis and how it is disrupted by mutations that affect cell proliferation and/or adhesion (some of the primary effects of Wnt pathway mutations found in colorectal cancer). In each case, we will compute the probability that a mutant population fixates in the crypt, and how this probability depends on the spatial location of the initial mutation in the crypt. The rest of the paper is organized as follows. In Section 2 we describe the mathematical model which is used to represent the colonic crypt and the simulations which we have performed. In Section 3 we present the results of these simulations, and we conclude in Section 4 with a summary of our findings and suggestions for future work. 


\section{Multiscale crypt model}

Our model of a colonic crypt has been described in detail previously by van Leeuwen et al. (2009); PittFrancis et al. (2009), we briefly re-introduce it here. We simulate the dynamics of individual cells, whose movement is governed by a model of mechanical interactions (Section 2.1). The proliferative state of each cell is governed by a cell-cycle model, which takes into account the local Wnt stimulus (Section 2.2).

\subsection{Mechanical model}

A variety of approaches can be used to model the mechanical behaviour of individual cells in a tissue. There are two basic components to any such model: the first step is to decide which cells are neighbours, and the second is to determine the forces transmitted by these neighbours. We adopt the tessellationbased, cell-centre approach of van Leeuwen et al. (2009), in which cells are defined by their centres. Cell movement is determined by assuming that each cell exerts a linear spring force on its neighbours. Neighbours are defined by a Delaunay triangulation of the cell centres, which is updated at each time step. Movement is assumed to be highly viscous or 'over-damped'; that is we neglect inertial terms. Balancing the linear spring forces on an individual cell, with a drag term representing cell-substrate adhesion, an equation of motion for the centre of each cell $i$ is gained,

$$
\frac{\alpha_{i} \eta}{\mu} \frac{\mathrm{d} \mathbf{r}_{i}}{\mathrm{~d} t}=\sum_{j \in S_{i}}\left(\left\|\mathbf{r}_{j}-\mathbf{r}_{i}\right\|-s_{i j}(t)\right) \frac{\left(\mathbf{r}_{j}-\mathbf{r}_{i}\right)}{\left\|\mathbf{r}_{j}-\mathbf{r}_{i}\right\|}
$$

where $\mathbf{r}_{i}$ denotes the position of the centre of cell $i, \mathbf{r}_{j}$ that of neighbouring cell $j, s_{i j}(t)$ is the equilibrium length of the spring connecting cells $i$ and $j$ at time $t, S_{i}$ is the set of cells that are adjacent to cell $i$ (in the Delaunay triangulation), $\alpha_{i}$ is a dimensionless scaling factor for mutant cells for the drag coefficient $\eta$, and $\mu$ is the spring constant. We take $\alpha_{i}=1$ for wild-type cells, and assume that all progeny of a given mutant cell share the same value $\alpha_{i} \equiv \alpha$, which we refer to hereafter as the adhesion parameter.

As noted by Meineke et al. (2001), the ratio $\eta / \mu$ can be written as a single parameter $\lambda$, which has units of time. A large value of $\lambda$ corresponds to a long timescale being associated with mechanical relaxation. We follow our previous study (van Leeuwen et al., 2009) by fixing $\lambda=120$ seconds in all simulations, as in wild-type crypts this results in cells migrating up the crypt over the experimentally observed time-scales. We use the crypt geometry introduced in our previous study (van Leeuwen et al., 2009), namely a fixed cylindrical domain

$$
\left\{(x, y) \in \mathbb{R}^{2}: 0 \leq x \leq c, 0 \leq y \leq h\right\}
$$

where $c$ and $h$ denote the circumference and height of the crypt respectively. A no-flux boundary condition is imposed at the base of the crypt, and cells that reach the top of the crypt are removed from the simulation, replicating sloughing. The average number of cells in histological sections, as given for the mouse large bowel by Sunter et al. (1979, Table 1, site (iii)), is approximately 16 cells in circumference and 20 cells in length. The simulated crypt has dimensions 14 'relaxed cell diameters' in circumference 
and 17 in height, thus we take $c=14$ and $h=17$. It should be noted that due to the increased proliferation (and therefore packing) in the base of the crypt we then obtain the appropriate number of cells in typical quasi-steady crypt cross-sections.

A forward Euler discretization of equation (1) is used to calculate the new cell locations, with a time-step of

$$
\Delta t=30 \times \min _{\forall i}\left(\alpha_{i}\right) \text { seconds, }
$$

(this was found to be sufficient to ensure numerical stability, notice it has to be decreased for cells with decreased adhesion $\alpha_{i}<1$ ). After each round of cell movements we progress each cell through its cell cycle, allowing cells to divide when necessary (see next section), removing cells when they reach above the top of the crypt, and recalculating cell neighbours by re-meshing. Further details on the implementation can be found in Mirams (2008) and Pitt-Francis et al. (2009).

\subsection{Cell-cycle model}

We have formulated a simple model of Wnt-dependent cell proliferation. Our crypts consist of proliferating and non-proliferating cells, the former encompassing stem and transit cells and the latter corresponding to differentiated cells (see Figure 1). Following van Leeuwen et al. (2009), we represent the Wnt gradient by a fixed external stimulus, whose non-dimensional concentration $W$ decreases linearly from 1 at the crypt base to 0 at the top of the crypt (Gregorieff and Clevers, 2005).

Near the base of the crypt, where cells are exposed to high levels of Wnt, we suppose the production of Wnt-dependent cell-cycle control proteins is enhanced and cells progress through the cell cycle. Near the crypt apex, where Wnt levels fall below a 'division threshold' $W_{\mathrm{thr}}$, cell division stops and cells are considered to be differentiated. We choose $W_{\text {thr }}$ so that the number of proliferative cells (and crypt turnover) in a healthy crypt matches experimental observations (Sunter et al., 1979; Wright and Alison, 1984b). For healthy cells the value of this division threshold is set to be $W_{\mathrm{thr}}=0.65$, so cells proliferate to $35 \%$ of the height of the crypt; we term this threshold height the proliferation ceiling, and denote it by $y_{\text {thr. }}$.

It is important to include an element of stochasticity into the cell cycle duration in order to prevent the unrealistic situation where all cells divide synchronously (as we will see, this would always happen, regardless of the initial conditions). Following Meineke et al. (2001), we assume that stochastic variation is a result of variation in the length of the $G_{1}$ phase only. Each proliferating cell is given a $G_{1}$ phase duration sampled from a normal distribution $N(9.4,1)$ hours (in practice, we truncate this distribution to ensure strictly positive times); with other cell cycle phases of $S=7.4 \mathrm{~h}, G_{2}=1.4 \mathrm{~h}$ and $M=0.72$ h (the cell cycle times from region 3 of mouse large bowel in Sunter et al., 1979). Differentiated cells are assumed to be in $G_{0}$ phase and never divide. There is no separate immortal population of stem cells, as this prevents the occurrence of monoclonal conversion (van Leeuwen et al., 2009; Fletcher et al., 2012). Instead, 'stem cells' are extrinsically defined by the high-Wnt micro-environment at the crypt base, rather than any internal property.

When a cell enters mitosis, as determined by its cell-cycle model, a new cell-centre is placed a distance 0.1 cell diameters away from the parent in a random direction. We do not include cell growth explicitly 
in the model; in order to prevent cells from springing apart abruptly when division occurs, we let the equilibrium length of the spring connecting parent and daughter cell increase linearly from 0.1 to 1 during the $M$ phase of the cell cycle. The equilibrium length of all other connected springs remains 1 during this time.

In order to study the effects of mutations affecting proliferation and cell-substrate adhesion independently, we decouple them by using simple models for each, as described below.

\subsection{Modelling changes to proliferation}

As noted in Section 1, certain mutations in the Wnt pathway enable proliferation to occur regardless of the level of the external Wnt stimulus (Sansom et al., 2007). Thus a disruption to the Wnt pathway may enable a mutant cell to continue to divide when exposed to a Wnt stimulus that inhibits proliferation in a wild-type cell. In this study we hypothesise that such disruption occurs to varying degrees according to the severity of the mutation. In more detail, we generate different mutant cells by varying the Wnt threshold $W_{\mathrm{thr}}$ that defines the proliferation ceiling $y_{\mathrm{thr}}$, allowing the latter to vary from $30 \%$ to $100 \%$ of the crypt height. Thus we use different height-dependent proliferation rules to investigate the role of spatially-dependent proliferation on the likelihood of a mutant population dominating a crypt.

\subsection{Modelling changes to adhesion}

Experimental results obtained by Sansom et al. (2004) indicate that mutations in the Wnt pathway influence cell migration. It is thought that this effect may be due to changes in cell-cell adhesion since $\beta$-catenin forms a bridge between adherens junctions and the cell cytoskeleton (Harris and Peifer, 2005).

Based on previous theoretical work (van Leeuwen et al., 2007), we hypothesise that the adhesion parameter $\alpha$ for mutant cells may differ from that of wild-type cells by up to ten-fold. In reality a ten-fold increase in $\beta$-catenin may not cause a correspondingly large increase in adhesion, as other factors such as the availability of E-cadherin could become rate-limiting. Thus our chosen range is likely to include (and extend beyond) those values of $\alpha_{i}$ that are physiologically realistic; we emphasize here that we are focusing on the qualitative effects of changes to adhesion on mutant behaviour.

\subsection{Implementation}

We have used Chaste, an open-source computational biology software library, to perform our simulations. The software implementation of the crypt model is discussed in Pitt-Francis et al. (2009), and the code used to generate the results presented in this paper is available as an open-source download from www.cs.ox.ac.uk/chaste.

Initially the crypt is set up with the dimensions discussed in Section 2.1 and allowed to evolve to a quasi-equilibrium state in which the mean number of cell divisions is equal to the mean number of cells sloughed from the crypt in any time period. In each of our simulations we ensure that the crypt is in such a quasi-equilibrium before introducing a mutant cell. 
Individual clones in each simulation are tracked as follows: each cell is initially assigned a unique 'ancestor index' (an integer denoting its initial node index in the mesh suffices for this); this label is passed to all of that cell's progeny; we then evolve the crypt until all cells in the crypt have the same ancestor index.

At this time the crypt has become monoclonal.

For simulations of mutant clones, we introduce mutant cells by randomly labelling a single cell in one of three height bands; in the bottom $5 \%$ of the crypt, in the bottom 5-10\% and in the bottom 10-15\% approximately corresponding to vertical cell positions 1, 2 and 3 (the reason no mutations are introduced higher than this will become clear in the results section). Mutant cells are given a particular proliferation ceiling $y_{\mathrm{thr}}$ (35\% of the crypt height for 'wild type' and between $30 \%$ and $100 \%$ of the crypt height for mutants with altered proliferation). Mutant cells are also given an adhesion parameter $\alpha$ of between 0.5 and 10 times that of wild type cells, as discussed above.

The simulation is runs until the mutant population is swept out of, or dominates, the crypt, and we record the time at which this happens. Examples of typical simulations in which a mutant cell is introduced are shown in Figure 2. In Figure 2(a) the mutant clone is 'swept out' of the crypt, whilst in Figure 2(b) the mutant clone 'dominates' the crypt. In this case the only difference between the simulations is the choice of initial labelled cell.

Due to the stochastic nature of the model, multiple simulations are needed to estimate the probability of a mutation taking over the crypt. If a simulation ends when a mutant population is swept out of the crypt, then we may re-use the final quasi-equilibrium state of the crypt as a starting point for our next simulation. However, if the simulation ends with mutant domination, then the cell density in the crypt may be higher than under wild-type conditions (especially if mutant cells have increased cell adhesion). Therefore, to obtain a new starting point for our next simulation, we re-use our first quasi-equilibrium state and evolve it for a random duration $(\sim \mathrm{U}(0,50)$ hours $)$ before introducing a mutant cell at random in the required height band.

We note that in order to build up statistical distributions, rather than simply show a 'typical' simulation result, the total amount of simulated 'crypt time' for this study was over $8 \times 10^{7}$ hours $(9,000$ years).

The number of realisations for each choice of parameter values accompanies each result.

\section{Results}

Given that the crypt model replicates experimentally observed patterns of proliferation, migration and turnover rates (van Leeuwen et al., 2009), we present some model predictions which follow from tracking its clones. We present results showing monoclonal conversion in wild-type crypts in Sections 3.1-3.3, and results for mutated cells in Sections 3.4-3.7.

\subsection{Monoclonal conversion always occurs}

In this section we study the process of monoclonal conversion by running multiple crypt simulations, as described in Section 2.5, in the absence of any mutation. In each case we track the progeny of each 

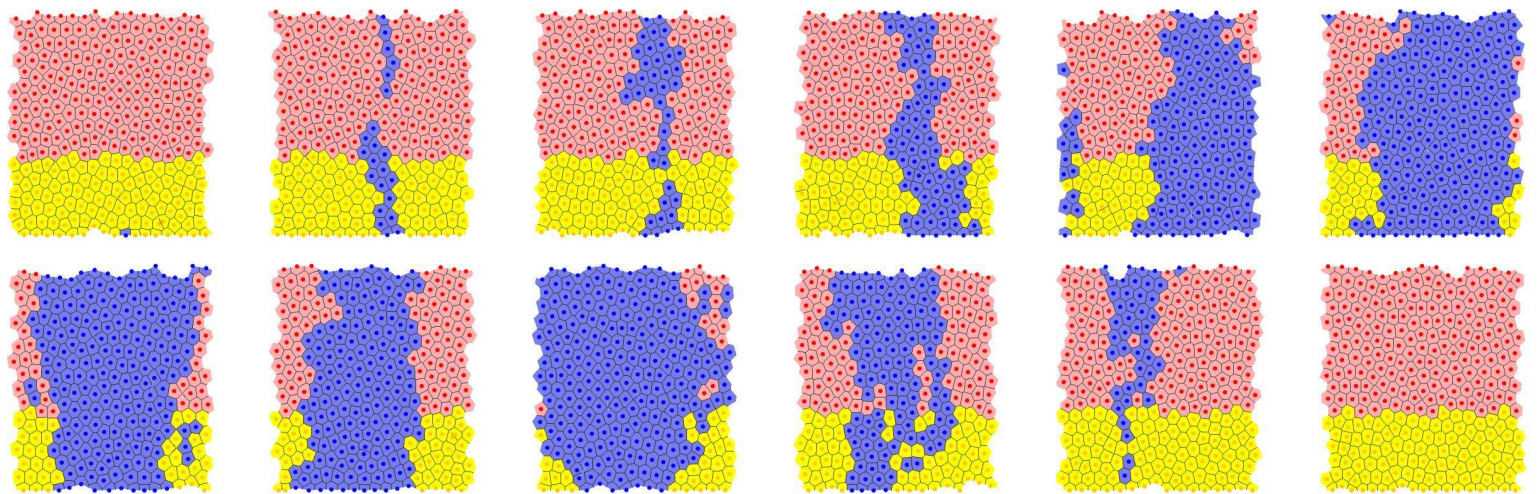

(a) A mutant population is swept out of the crypt,
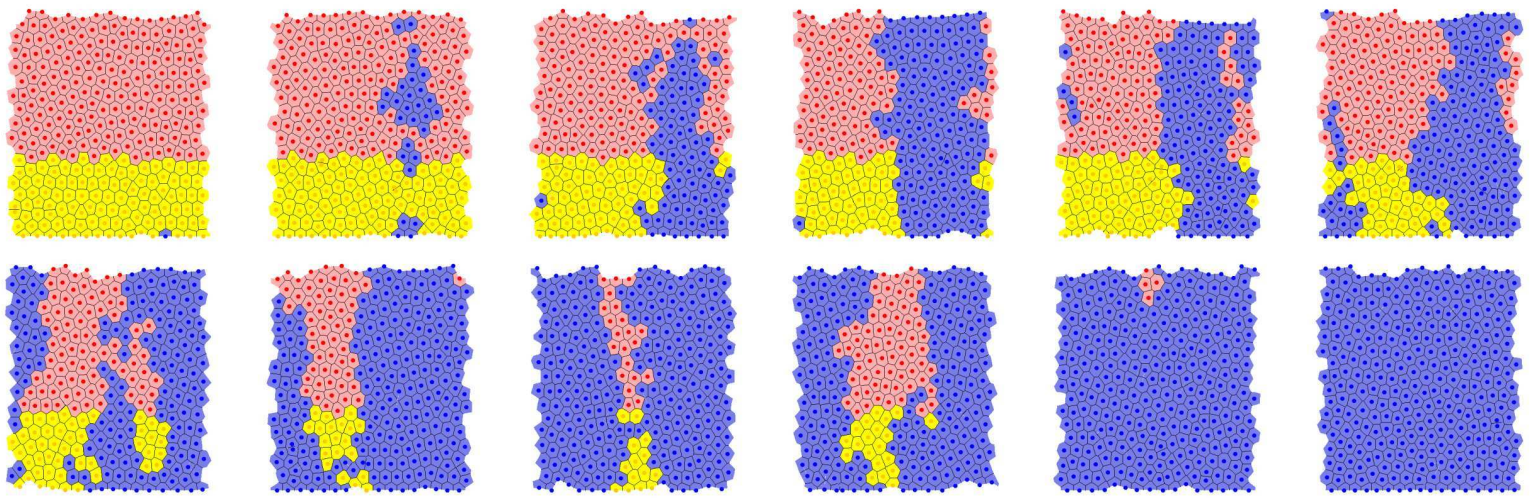

(b) A mutant population dominates the crypt.

Figure 2: Evolution of a mutant population within a crypt. A single mutant cell is introduced into the base of a crypt in quasi-equilibrium. Snapshots are shown for every 100 hours $(\sim 4$ days or crypt renewal time) thereafter, going left to right then top to bottom, with the final snapshot at 1200 hours (50 days). Here the 'mutant' population retains the same adhesion parameter $(\alpha=1)$ and proliferative ceiling $\left(y_{\mathrm{thr}}=35 \%\right)$ as healthy cells, and thus the mutation may be thought of as a neutral label. Red cells are differentiated, yellow cells are proliferating transit cells, and blue cells denote both proliferating and differentiated cells in the mutant clonal population. Domination such as that shown in (b) occurred in $500 / 10129$ simulations in a 'wild-type' crypt. 
initial cell and stop the simulation as soon as the crypt becomes monoclonal, and all cells in the crypt have the same ancestor.

In Figure 3 we present a histogram from 4000 simulations summarising the times at which the crypts first become monoclonal. We observe that in each case, given sufficient simulation duration, monoclonal conversion always occurs. The time taken for this to occur did not exceed 6,040 simulated hours $(\approx 36$ weeks) for the 4000 simulations we performed. This result is consistent with the recent experimental and theoretical demonstration by Lopez-Garcia et al. (2010) that the cell population at the base of the crypt follows a pattern of neutral drift dynamics, whereby the loss of a cell from the proliferating compartment is compensated by the division of a neighbour. This process ensures a population that is of constant size on average, and leads to ever fewer yet larger clonal populations and a drift toward monoclonality (Snippert et al., 2010).
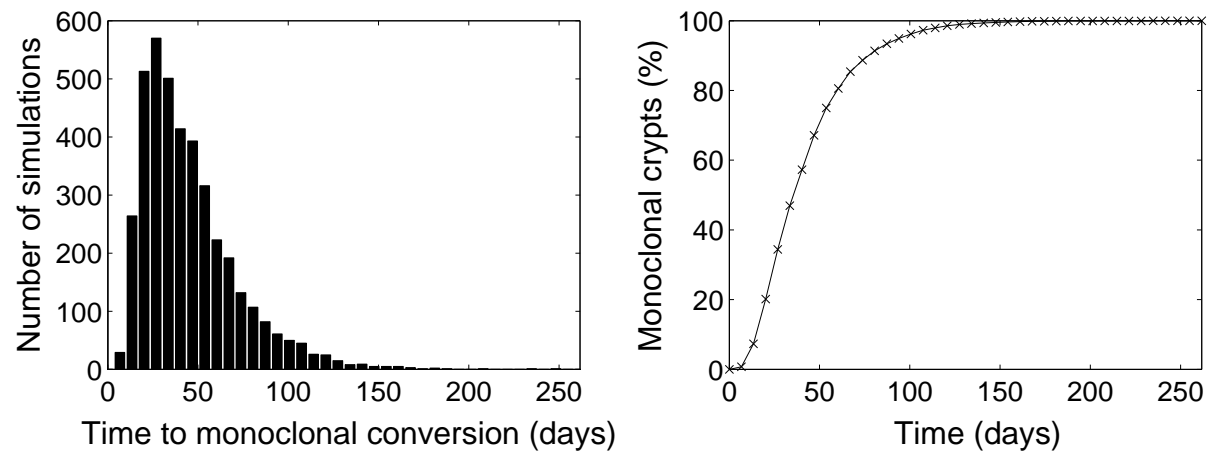

Figure 3: Time to monoclonal conversion. Left: as a histogram, Right: as a cumulative distribution function. Results shown are from 4000 simulations of a wild-type crypt, which always becomes monoclonal. Parameter values for these (and following) simulations are as discussed in Sections 2.1 and 2.2

\subsection{There are no immortal stem cells}

The process of monoclonal conversion does not require that the cell which gave rise to the entire population remains in the crypt. We used data from the 4000 simulations described above to predict the time at which the original ancestor cell (the cell which at $t=0$ contained the 'ancestor index') is swept out of the crypt. In Figure 4 we present a histogram and cumulative distribution function for the time at which the original ancestor cell that led to the dominant clone is lost from a crypt. We remark that the timescale over which this process occurs is faster than that for monoclonal conversion. In these simulations, the ancestor cell was always expelled from the crypt before it became monoclonal.

\subsection{Dominant clones originate at the base of the crypt}

In this section we introduce a 'neutral' mutant (or 'labelled' clone), with the same properties as the wild-type cells, such as those shown in Figure 2, by simply labelling an existing cell and tracking its progeny. Each simulation ends when the labelled clonal population dominates or is swept out of the crypt. We performed 12129 simulations, as described in Section 2.5, in order to obtain 500 for which the labelled population eventually dominates the crypt. 

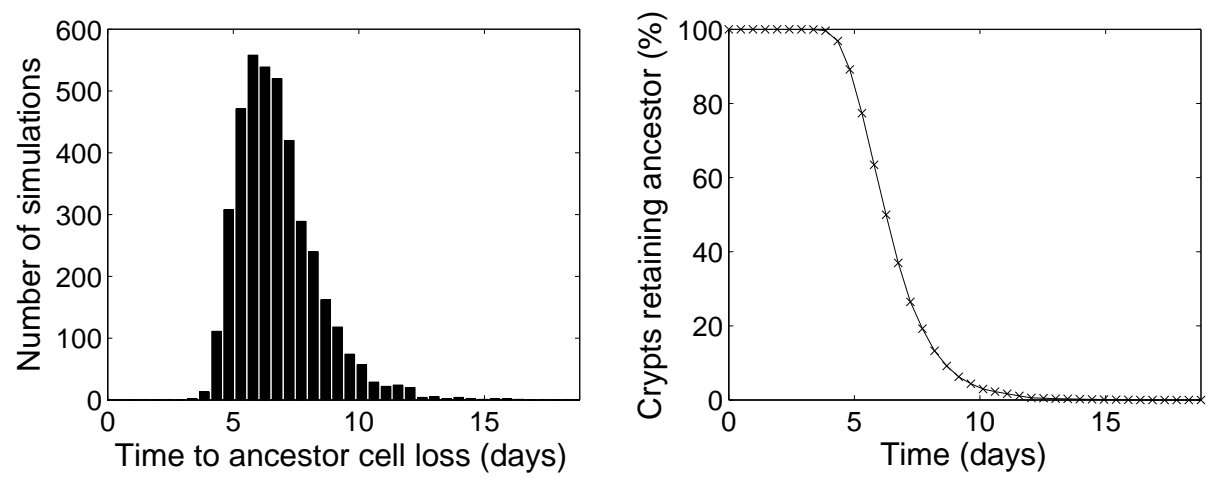

Figure 4: Left: histogram of times at which the ancestor cell leaves the crypt, Right: Times as a cumulative probability function. Results shown are from 4000 simulations of a wild-type crypt.

Figure 5 summarises these results and shows how the probability that a labelled clone becomes dominant varies as we vary the distance from the crypt base at which the label is introduced. At the base of the crypt the likelihood of becoming the dominant clone approaches $6 \%$, which (as one might expect) is approximately equal to $1 / N$, where $N$ is the number of cells at the crypt base. Labels introduced elsewhere in the crypt are quickly swept out: in over 12,000 simulations none of these neutral mutations introduced above $10 \%$ of the height of the crypt ever dominated. Figure 6 shows a histogram of the duration of these simulations.

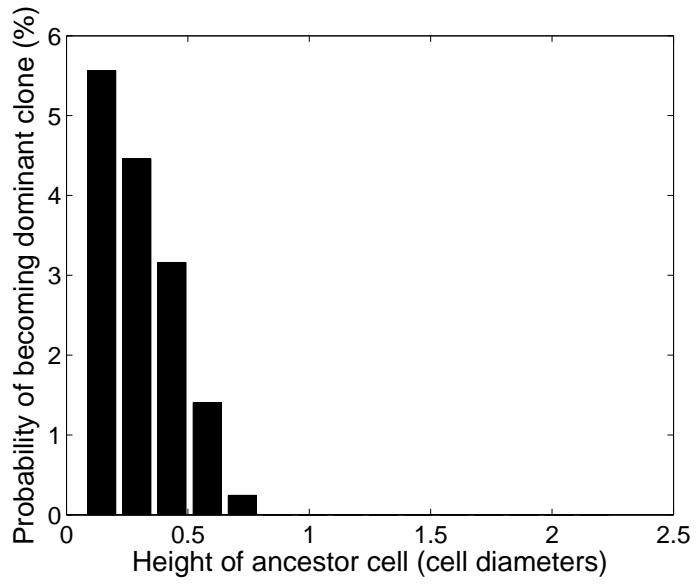

Figure 5: Probability of the progeny of a cell labelled with a neutral mutation becoming the dominant clone as a function of height up the crypt. Results were generated from 12, 129 simulations, of which 500 resulted in the labelled population dominating the crypt.

We see that the vast majority of simulations end when the mutant population is swept out of the crypt within 10 days of the initial mutation. Those mutants which dominate do so over a significantly longer period of time, up to 140 days after the initial mutation. In the case of longer simulations, in which the mutant population remains in the crypt for an extended period of time, the number of competing clones is typically reduced to 2 , and for a 'harmless' mutation the probability of domination is approximately $50 \%$. In such cases, the distribution of times for dominant clones to take over the crypt follows that shown in Figure 3. This is in agreement with previous theoretical work by Fletcher et al. (2012). Thus we observe roughly equal numbers of 'loss' and 'dominance' for simulations ending at high durations. 


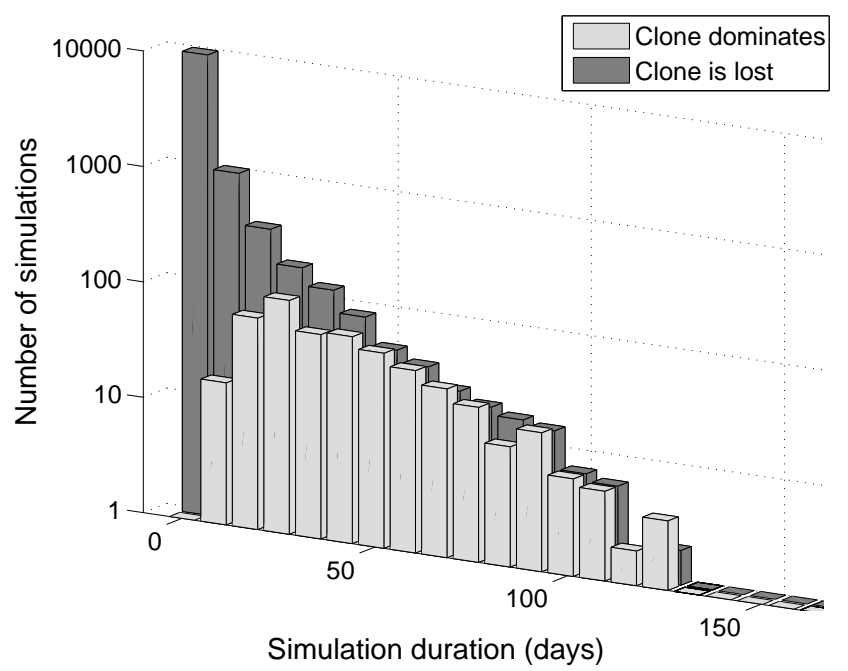

Figure 6: Histogram of the durations of crypt simulations in which a neutral mutation is introduced. Simulations are run until the mutant population is 'lost' from (swept out of) or 'dominates' the crypt. Results were generated from 12129 simulations, of which 500 resulted in the labelled population dominating the crypt. Note that a logarithmic (base 10) scale is used for the number of simulations.

These observations, in a wild-type setting, suggest that monoclonal conversion should not be thought of as a 'one-off' event, but rather as a continuous process. The clonal population giving rise to the entire population continuously shifts, in the battle to occupy the base of the crypt. In the following sections we use our model to investigate how different adhesive or proliferative properties associated with a particular mutation might accelerate this process.

\subsection{Effect of varying mutant adhesion parameter}

In Figure 7 we show how the likelihood of a mutant clone becoming dominant in the crypt depends on its adhesion parameter $\alpha$. As one might expect, mutant clones with increased cell-substrate adhesion are more likely to dominate. This is because in equation (1) the viscous drag force associated with adhesion is inversely proportional to velocity. The more adhesive mutant clones therefore move more slowly than the wild-type cells with which they are competing for space, although they proliferate at the same rate. For low and intermediate levels of mutant cell-substrate adhesion, the associated advantage depends approximately linearly on the adhesion parameter, this relationship is discussed further in Section 3.6.

\subsection{Effect of varying mutant proliferation ceiling}

As described in Section 2.2, cells are assumed to proliferate between the base of the crypt and a given proliferation ceiling $y_{\mathrm{thr}}$, set to be $35 \%$ of the height of the crypt for wild-type cells. We performed monoclonality simulations for mutant cells, varying $y_{\mathrm{thr}}$ in the range $30 \%$ to $100 \%$ of the height of the crypt. In Figure 8 we present the results, showing how the likelihood of a mutant clone taking over the crypt changes as $y_{\text {thr }}$ varies in this range.

One might expect the probability of domination to increase monotonically as the proliferation ceiling increases, but, as noted in Section 3.3, only those mutations occurring within one or two cell diameters 


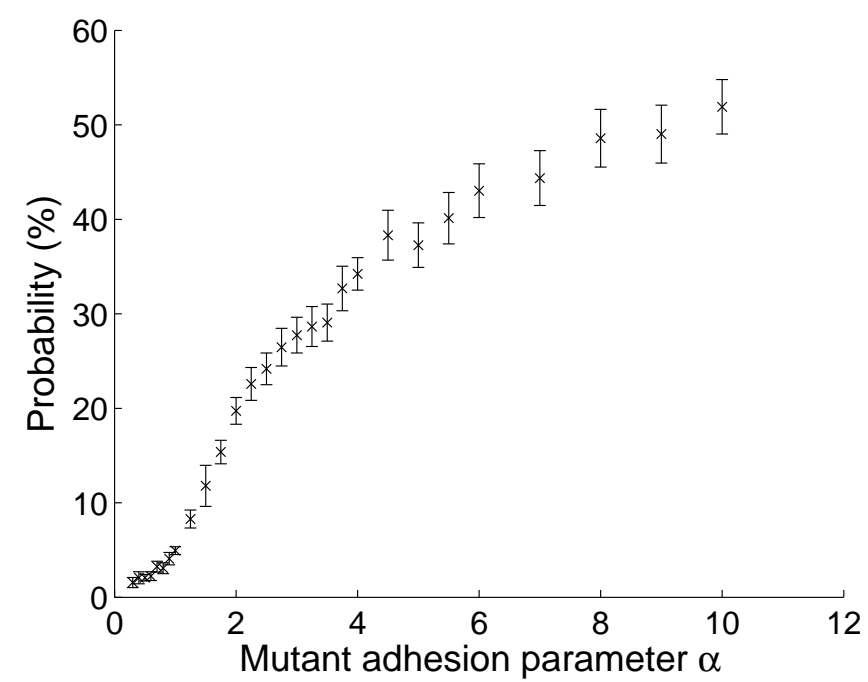

Figure 7: Probability of a mutation introduced at the base of the crypt becoming the dominant clone, as the clone's adhesion parameter $\alpha$ is varied from 0.3 to 10 . In these simulations the mutation is assumed to affect cell-substrate adhesion but not proliferation $\left(y_{\mathrm{thr}}=35 \%\right)$. Error bars denote the $95 \%$ confidence interval for a binomial distribution probability $p$, according to $p \pm z_{0.975} \sqrt{p(1-p) / n}$, where $n$ is the number of simulations (600 for each value of $\alpha$ ) and $z_{0.975}$ is the 97.5 th percentile of a standard normal distribution.

of the crypt base can become established. In the simulations considered in the previous section, the difference between wild-type and mutant cell behaviour is only apparent some distance up the crypt. Therefore we may expect $y_{\text {thr }}$ to have little effect on a mutant clone's probability of domination. However, as Figure 8 shows, we instead find that the probability of domination increases as $y_{\text {thr }}$ increases in the range $30 \%<y_{\mathrm{thr}}<60 \%$, and attains a maximal value when $y_{\mathrm{thr}} \approx 50 \%$, before asymptotically decreasing to a value of around $9 \%$ at $y_{\mathrm{thr}}=100 \%$.

To investigate this behaviour further we repeated our simulations, separating them into two groups: those in which the mutant population dominates the crypt and those in which it is swept out. We recorded the mean vertical component of forces per unit time experienced by cells at the base of the crypt, averaged over all simulations in each group. These results are presented in Figure 9 and reveal the influence of cells proliferating higher up the crypt: the increased levels of proliferation introduce more cells into the crypt. This creates a higher cell density and therefore larger forces between cells. These forces propagate to the base of the crypt, since this is the only fixed boundary against which cells may 'push'. In simulations where the mutant clone dominates, mutant cells at the crypt base experience a higher mean vertical force than wild-type cells, which acts to keep the mutant cells in the stem cell niche'. In simulations where a wild-type clone dominates, the reverse is true. However, note that in both simulation groups, the mean vertical force on cells at the base of the crypt increases in magnitude as the proliferation ceiling $y_{\mathrm{thr}}$ increases.

Based on these results, we postulate the following mechanism to explain the results presented in Figure 8. When a mutant clone continues to proliferate to heights slightly above those of wild-type cells, increased proliferation occurs directly above the mutant clone's position on the crypt base, and bestows a competitive advantage upon it by 'pushing it down'. By contrast, when a mutant clone proliferates 


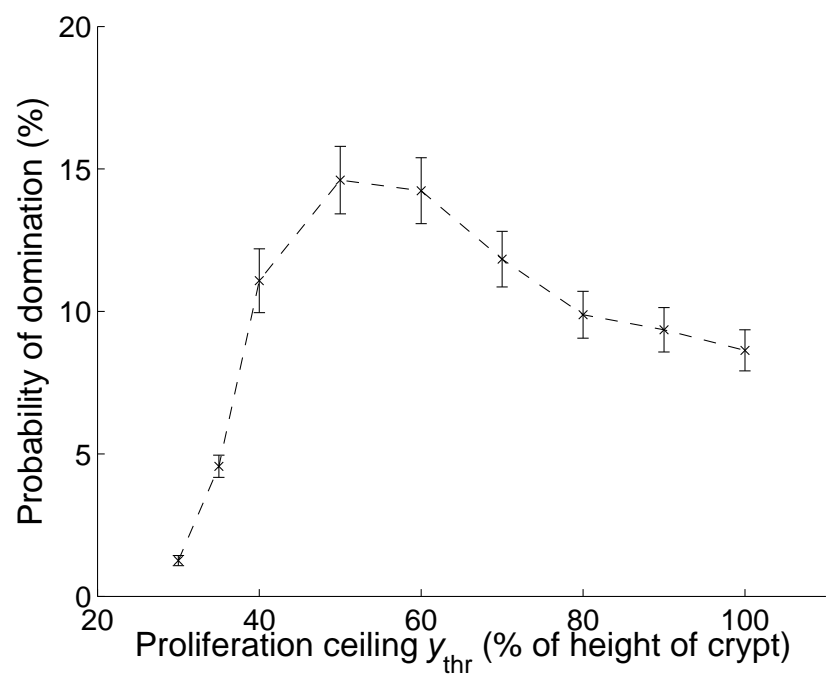

Figure 8: Probability of a mutation introduced at the base of the crypt becoming the dominant clone, as the clone's proliferation ceiling $y_{\text {thr }}$ is varied. In these simulations the mutation is assumed to affect proliferation but not cell-substrate adhesion $(\alpha=1)$. Error bars denote the $95 \%$ confidence interval for a binomial distribution probability $p$, according to $p \pm z_{0.975} \sqrt{p(1-p) / n}$, where $n$ is the number of simulations and $z_{0.975}$ is the 97.5 th percentile of a standard normal distribution. Variable numbers of simulations were performed until 500 dominations occurred (at least 3000 simulations) for each proliferation ceiling.
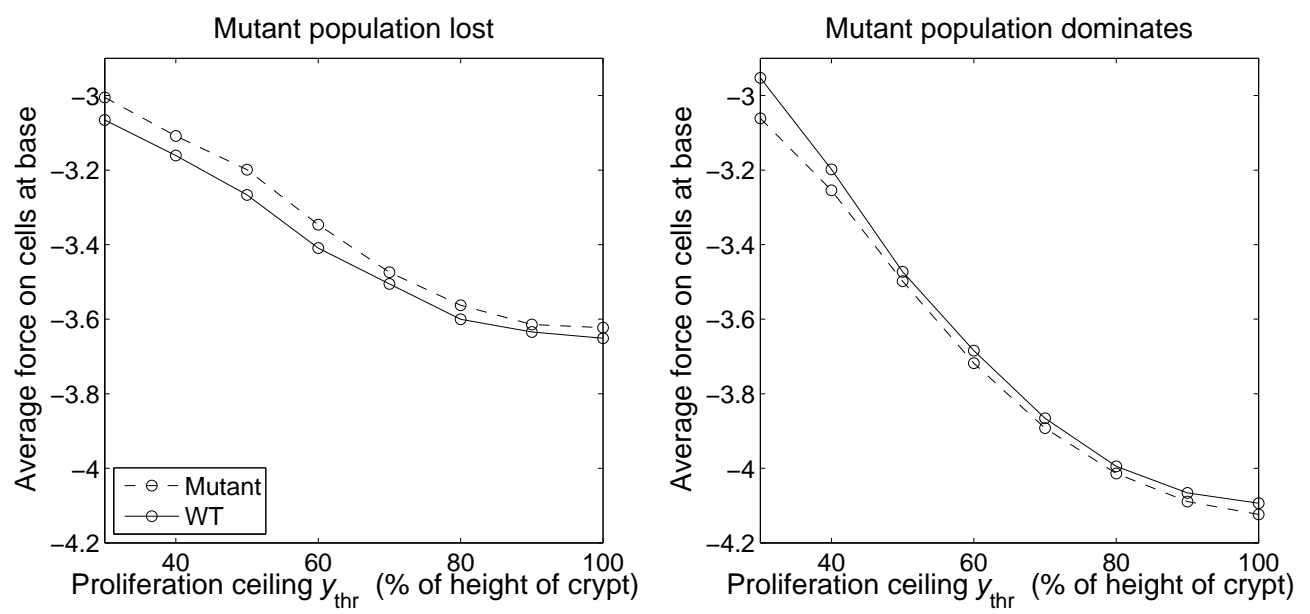

Figure 9: Left: mean vertical component of the force per unit time on cells at the base of the crypt in simulations in which a mutant population with altered proliferation ceiling $(\alpha=1)$ dominates the crypt, where the average is taken over 500 simulations for each value of the proliferation ceiling $y_{t h r}$. Right: mean vertical component of the force per unit time on cells at the base of the crypt in simulations in which a mutant population with altered proliferation ceiling $(\alpha=1)$ is swept out of the crypt, where the average is taken over a variable number of simulations (at least 2500) for each value of $y_{t h r}$. 


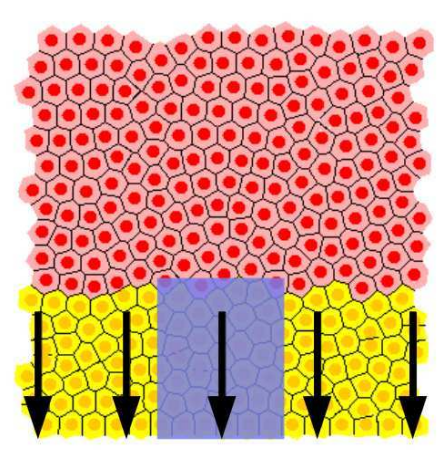
in Figure 10. throughout the crypt, the clone begins to spread laterally in the upper portion of the crypt where the wild-type cells do not proliferate. The resulting increased downward vertical forces act upon both the mutant cells, and a portion of the wild-type cells at the crypt base, providing a strong advantage not only to the mutant cells, but also to nearby wild-type cells. A schematic of this mechanism is depicted
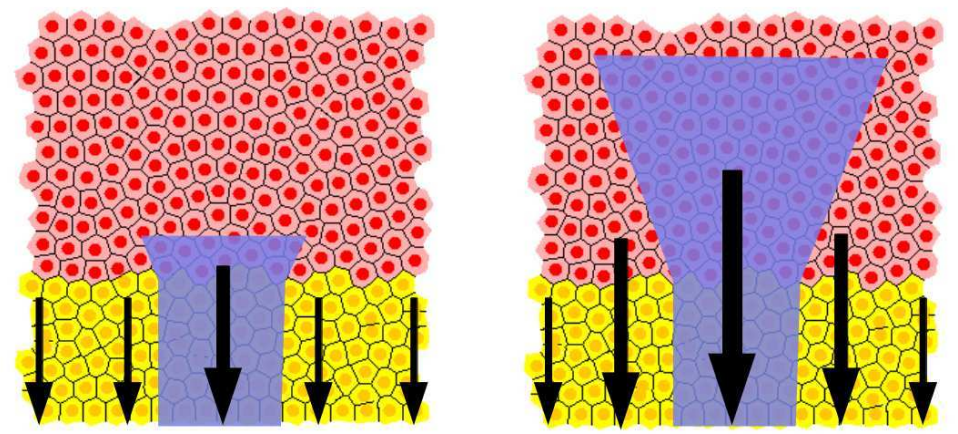

Figure 10: A schematic of the mechanism by which proliferative ceiling relates to probability of domination, with size of forces (generated by proliferation and increased cell density) represented by size and thickness of arrow. Left: mutant clone proliferates to same height as wild-type cells. Middle: mutant clone proliferates higher than wild-type cells, and causes an increased vertical force above the mutant clone. Right: mutant clone proliferates to the top of the crypt, spreads laterally and causes an increased vertical force on the mutant clone and its wild-type neighbours. Note that the areas shaded blue represent average behaviour of a mutant clone, rather than the result of a single simulation.

\subsection{Comparison with simplified one-dimensional model}

To investigate further the relationship between the mutant adhesion parameter $\alpha$, proliferation ceiling $y_{\text {thr }}$ and probability of crypt domination, we may compare our results to those obtained using a simple stochastic model that tracks the dynamics of a mutant cell introduced at the crypt base. This model is based on the work of Komarova (2007), and is a one-spatial-dimension generalization of a mass-action Moran birth-death process. We note that a similar modelling framework has been used by Nowak et al. (2003) to understand the effect on the rate of somatic evolution of a linear spatial architecture. Here we are are interested in the circumference of the base of the crypt, since we have already established that the probability of dominant mutations occurring above this region is negligible. We shall assume for simplicity that once a mutant population occupies the entirety of the bottom row of the crypt, it will dominate the crypt with probability one.

We assume that cells in the bottom row of the crypt are aligned along a regular grid, at locations $1, \ldots, N$ (where $N \approx 16$, as discussed in Section 2.1). We impose periodic boundary conditions, so that locations 1 and $N$ are neighbours, and make the simplifying assumption that the number of cells in this row remains constant over time. We assume that cells are removed at random from this row, due to mitotic forces propelling them up to the next row. Whenever a cell is removed, a division event occurs, with a daughter cell being generated by one of the cells that was adjacent to the removed cell.

We assume that there is an initial mutant cell, which produces mutant progeny. As in the rest of this study, we assume that no other mutation occurs over the timescale of interest. As we are interested in 
the probability of mutant domination, we only need take into account those changes in configuration that affect the number of mutant cells. Note that in this model, a mutant colony that originated as one cell can only occupy adjacent positions; in the cell-based model this is not enforced, but is usually the case. We let $P_{\exp }^{\mathrm{l}}$ and $P_{\mathrm{exp}}^{\mathrm{r}}$ denote the probabilities that a cell removal/division event results in a mutant population (of size greater than one) expanding to the left and right respectively, and let $P_{\text {con }}^{1}$ and $P_{\text {con }}^{\mathrm{r}}$ denote the probabilities that the population contracts to the left and right respectively. We also let $P_{\text {ext }}$ denote the probability that a mutant population of size one becomes extinct following a cell removal/division event. The mutant population differs from the wild-type population in its relative fitness $(r)$. This relative fitness determines how much more likely a mutant population is to expand to fill a gap left by an extinction, than the wild-type population is. That is the probabilities of expansion, contraction and extinction are given in terms of $r$ and $N$ by

$$
\begin{aligned}
& P_{\mathrm{exp}}^{\mathrm{l}}=P_{\mathrm{exp}}^{\mathrm{r}}=\frac{1}{N} \frac{r}{1+r}, \\
& P_{\mathrm{con}}^{\mathrm{l}}=P_{\mathrm{con}}^{\mathrm{r}}=\frac{1}{N} \frac{1}{1+r}, \\
& P_{\mathrm{ext}}=\frac{1}{N} .
\end{aligned}
$$

As discussed by Komarova (2007), periodicity means that the probability of mutant domination does not depend on the location of the mutant population, but only on its size. Thus we may derive the following system of equations for the probabilities $\pi_{i}$ that the mutant population will dominate the bottom row starting from an initial patch of $(i+1)$ mutant cells:

$$
\begin{aligned}
\left(P_{\mathrm{exp}}^{\mathrm{r}}+P_{\mathrm{con}}^{\mathrm{r}}\right) \pi_{i} & =P_{\mathrm{exp}}^{\mathrm{r}} \pi_{i+1}+P_{\mathrm{con}}^{\mathrm{r}} \pi_{i-1}, \quad 0<i<N-1, \\
\left(P_{\mathrm{exp}}^{\mathrm{r}}+\frac{1}{2} P_{\mathrm{ext}}\right) \pi_{0} & =P_{\exp }^{\mathrm{r}} \pi_{1}, \\
\pi_{N-1} & =1 .
\end{aligned}
$$

Solving this linear system, we deduce that $\rho(r)$, the probability of fixation starting from a single mutant cell, is given by

$$
\rho(r) \equiv \pi_{0}=\frac{2 r^{N-1}(1-r)}{1+r+r^{N-1}-3 r^{N}} \rightarrow \frac{1}{N} \text { as } r \rightarrow 1
$$

$$
\begin{aligned}
& \text { The dependence of } \rho \text { on fitness } r \text { is shown in Figure } 11 \text {. Note there is a monotonic and saturating increase } \\
& \text { in the probability that a mutant dominates the base of the crypt as } r \text { increases. } \\
& \text { Whilst simplified models are more amenable to mathematical analysis, it is often not immediately clear } \\
& \text { how to relate their parameters to experimentally accessible quantities. The results of our cell-based } \\
& \text { model simulations offer an opportunity to examine what relationship, if any, there may be between the } \\
& \text { mutant fitness } r \text { in this simplified model and the two physical properties that vary for mutants in our } \\
& \text { cell-based model, namely the adhesion parameter } \alpha \text { and proliferation ceiling } y_{\text {thr }} \text {. } \\
& \text { In Figure } 12 \text { we plot the values of } r \text { that result in the mean domination probabilities observed in the } \\
& \text { previous sections when } \alpha \text { and } y_{\text {thr }} \text { vary. In each case we also plot a best-fit linear relationship between } \\
& \text { these data. As Figure } 12(\text { a) shows, there is strong evidence of a linear relationship between } r \text { and } \alpha \text { for }
\end{aligned}
$$


the range of values considered in the simulations. This implies that in this simplified model, the concept of relative fitness can be used to describe the behaviour resulting from a mutant population with altered adhesion. In contrast, as shown in Figure 12(b), no clear relationship exists between relative fitness at the crypt base and $y_{\mathrm{thr}}$. We infer that in the case of altered proliferation the two-dimensional geometry of the crypt must be taken into account, when attempting to describe the relative fitness. The behaviour resulting from increased adhesion in the two-dimensional model appears to be captured well by increased fitness of the mutants at the crypt base.

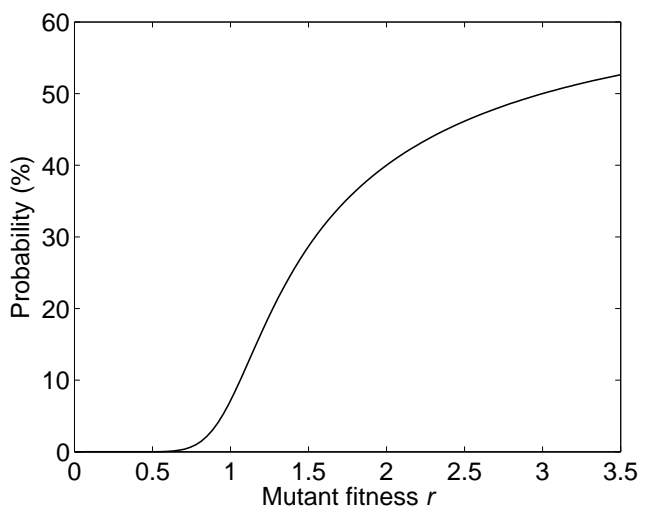

Figure 11: Probability of a mutation introduced at the base of the crypt becoming the dominant clone, as a function of the clone's fitness $r$ for $N=16$, according to a simpler stochastic model based on work by Komarova (2007).

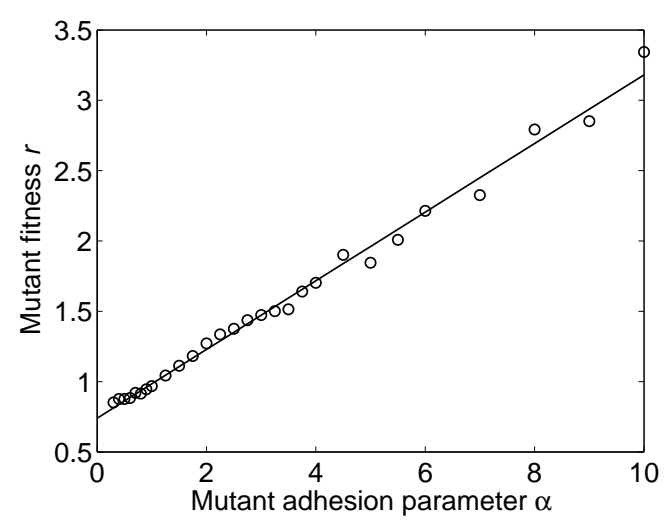

(a) fitness and mutant adhesion $\alpha$

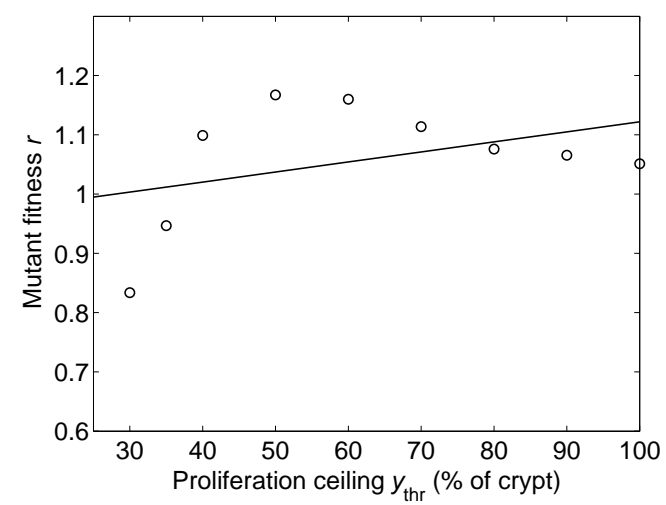

(b) fitness and proliferation ceiling $y_{\text {thr }}$

Figure 12: Relationship between clone fitness $r$, and the increase in domination probability seen in our cell-based simulations (Figures 7 and 8). The straight line fit in (a) is $r=0.740+0.244 \alpha$, in (b) $r=0.945+0.00181 y_{\text {thr }}$.

\subsection{Effect of varying mutant adhesion parameter, proliferation ceiling and height of mutation}

Having investigated the effect of varying in turn each of the three mutant properties (height of the initial mutation, adhesion parameter and proliferation ceiling), in this section we consider how the probability of domination varies when a mutation affects all three properties.

It is not computationally feasible to perform an exhaustive sweep of this three-dimensional parameter space. Therefore, guided by our findings in the previous sections, we restrict attention to certain values for 


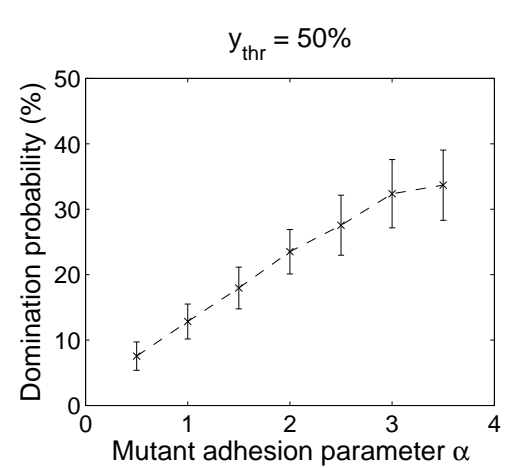

each parameter. For the adhesion parameter $\alpha$ we consider variation from 0.5 to 3.5. For proliferation ceiling $y_{\mathrm{thr}}$, we examine four cases (wild-type 35\%,50\%, 90\% and 100\% of the height of the crypt) chosen as they correspond to the heights to which common mutations in the Wnt pathway are expected to proliferate [in earlier theoretical work (van Leeuwen et al., 2009)], and covered extreme points on Figure 8. For the height of the initial mutation we consider the stratifications as above (for $<5 \%, 5-10 \%$ and $10-15 \%$ of the height of the crypt). For all combinations of parameters the $10-15 \%$ band did not yield any clones that dominated the crypt, and hence these results are not shown.
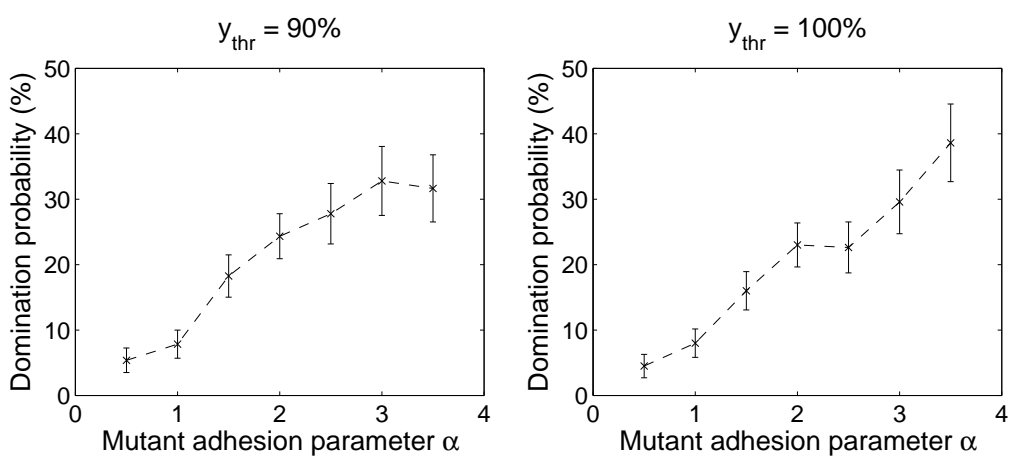

Figure 13: Series of graphs showing how the probability that a mutation introduced at the base of the crypt becomes the dominant clone, varies with the clone's adhesion parameter, $\alpha$. Error bars denote the 95\% confidence interval for a binomial distribution probability $p$, according to $p \pm z_{0.975} \sqrt{p(1-p) / n}$, where $n$ is the number of simulations (600 for each value of $\alpha$ ) and $z_{0.975}$ is the 97.5 th percentile of a standard normal distribution. Results for proliferation up to the following proportions of the crypt, left: 50\%; middle: 90\%; and right: 100\%. Results for 35\% (wild type) are shown in Figure 7 .

Figure 13 shows how the probability of domination varies with adhesion parameter for mutations introduced at the crypt base with different proliferation ceilings. We see that this probability is most sensitive to changes in adhesion, with the overall monotonic dependence on $\alpha$ being preserved for all values of $y_{\text {thr }}$ considered. We would anticipate from Figure 8 that those mutations with $y_{\mathrm{thr}}=50 \%$ will have a larger probability of domination than the other mutations considered, and this is indeed observed (for any given value of the adhesion parameter $\alpha$ ) in Figure 13.

Figure 14 shows how the probability of mutant domination varies with the height at which the initial mutation occurs, for different values of the mutant proliferation ceiling and adhesion parameter. In the case of normal cell-substrate adhesion $(\alpha=1)$, we observe that the probability of domination for a mutation occurring higher up the crypt increases with proliferation ceiling, in agreement with the results shown in Figure 8. At a higher level of mutant cell-substrate adhesion $(\alpha=2.0)$, there is a slight increase in the probability of domination for mutations occurring higher up the crypt. This coincides with an increase in the duration of time for which a mutant clone persists in the crypt prior to becoming dominant or being swept out.

In Figure 15 we present histograms of the time taken for successful mutants with altered cell-substrate adhesion and proliferation to dominate the crypt. Using the same combinations of $\alpha$ and $y_{\text {thr }}$ as those considered in Figure 14, we find that the time taken for domination to occur increases slightly with $y_{\text {thr }}$ when $\alpha=1$, and more markedly when $\alpha=2$. Thus the combination of higher cell-substrate adhesion 

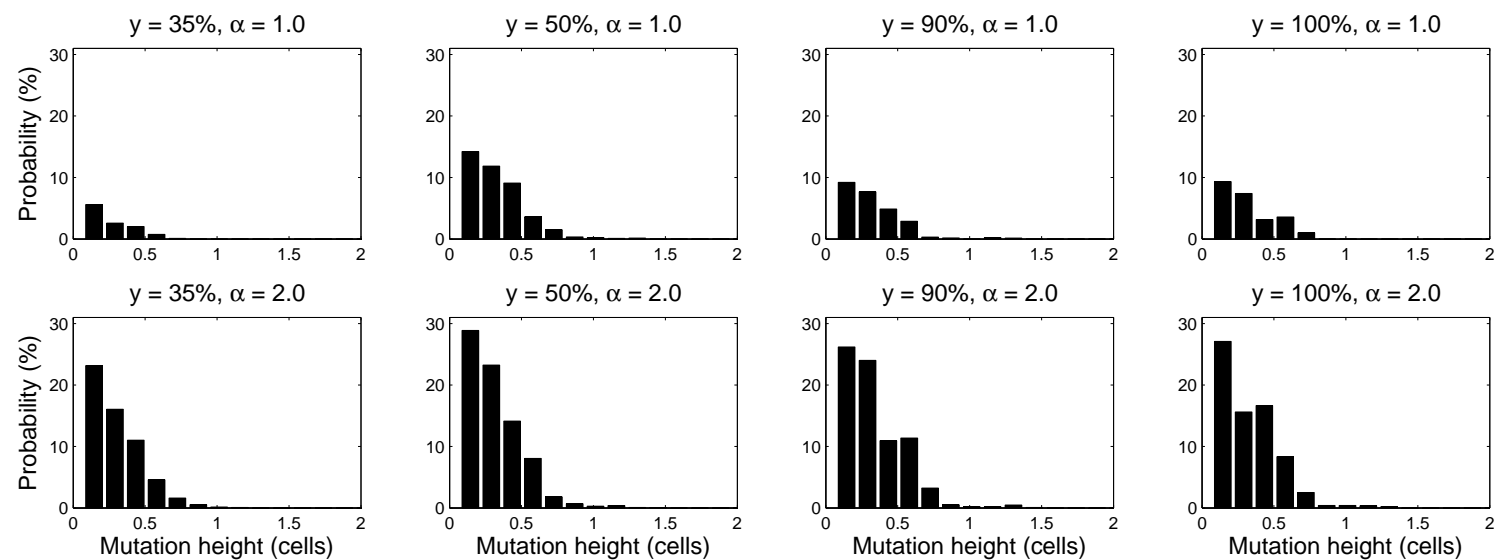

Figure 14: Probability of a clone dominating the crypt, for varying choices of the initial mutation height. Results shown for four values of $y_{\mathrm{thr}}(35,50,90$ and $100 \%$ of the height of the crypt) and for two different values of $\alpha$ (1.0 and 2.0$)$. Each plot summarises the results from at least 350 simulations with dominant mutant clones, and a corresponding proportion of simulations where the mutant clone is eliminated from the crypt.

and greater vertical downward forces arising from a higher proliferation ceiling (as shown in Figure 9) results in a longer period of competition before a successful mutant takes over the crypt.

\section{Discussion}

Intestinal homeostasis requires a coordinated programme of cell proliferation, migration and differentiation. The initiation of most colorectal cancers involves genetic alterations resulting in 'over-activation' of the Wnt signalling pathway. These alterations disrupt normal patterns of proliferation and cell-cell adhesion. Identifying the mechanisms by which a mutant population may colonize a crypt is therefore essential to understanding the origins of colorectal cancer.

In this article we have undertaken a computational study of how phenotypic variations, represented by differences in the proliferative and adhesive properties of individual cells, affect the likelihood that the progeny of a mutated cell will dominate a colonic crypt. We have employed a spatial model of a colonic crypt that distinguishes individual cells. The model couples the cell cycle and cell division with the mechanics of cell movement. By performing multiple simulations, varying not only the properties of the mutant cells but also the spatial location of the initial mutation, we have used the model to predict how the probability of domination depends on the properties of the mutant cells.

We began by considering the process of monoclonal conversion by running multiple crypt simulations in the absence of any mutation and, in each case, tracking the time taken for the progeny of one of the cells initially present to take over the entire crypt. Given sufficient time, monoclonal conversion always occurs in our model, since those cells that undergo symmetric division are following a pattern of neutral drift, as detailed by Snippert et al. (2010). A prediction of our model is that monoclonal conversion does not require that the cell that gave rise to the entire population remains in the crypt. Indeed, in our simulations the timescale over which the original ancestor cell is lost from the crypt was found to be significantly shorter than that over which monoclonal conversion occurred. 

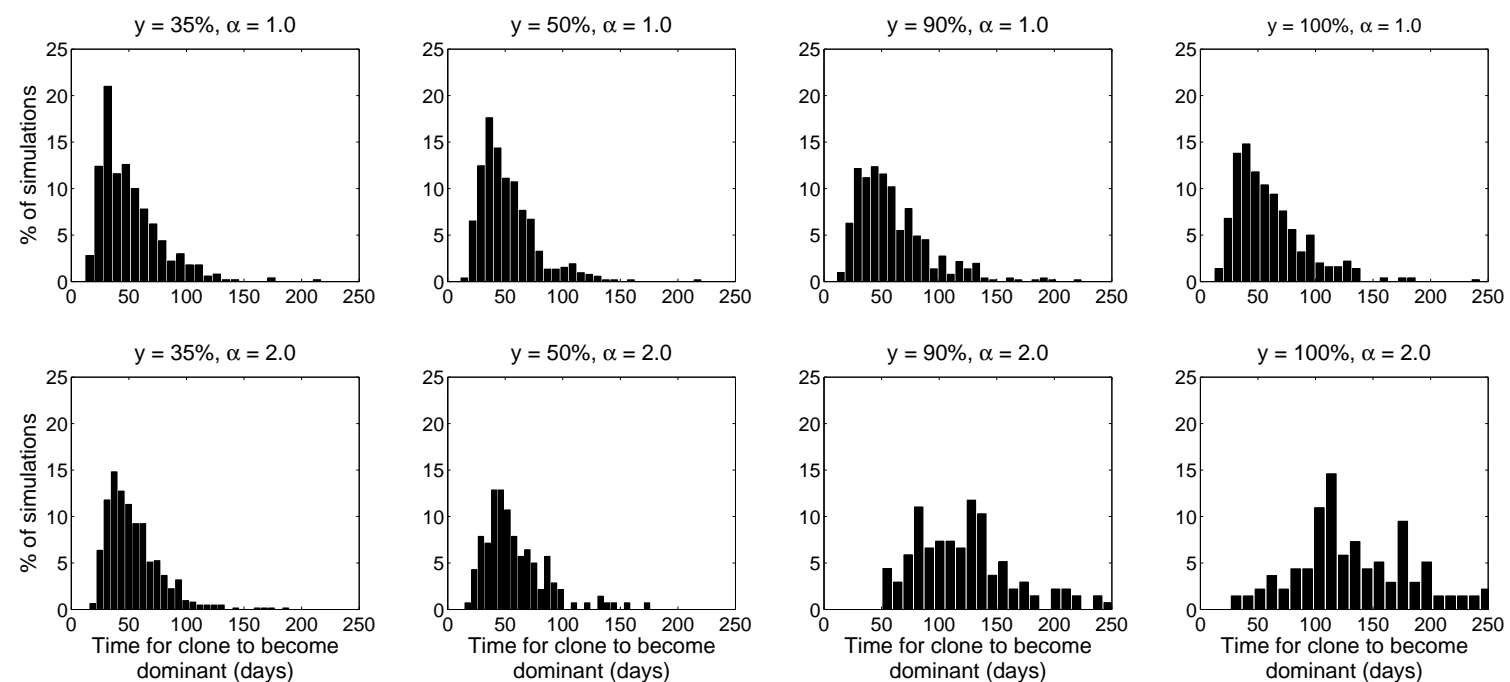

Figure 15: Histograms of the duration of a dominant clone's persistence in the crypt. Results shown for four values of $y_{\mathrm{thr}}$ $(35,50,90$ and $100 \%$ of the height of the crypt) and for two different values of $\alpha$ (1.0 and 2.0). Each plot is generated from at least 350 simulations in which a mutant population, with given proliferation ceiling and adhesion parameter, dominates the crypt.

We observed that the ability of a mutant clone to take over a crypt is extremely sensitive to the height at which the mutation occurs, with most mutants being swept out of the crypt due to normal turnover and flux of cells up the crypt axis. In particular, 'neutral' mutations introduced more than one or two cells above the base of the crypt were unable to dominate it.

The probability of domination was found to be strongly affected by the extent to which mutations alter cell-substrate adhesion, represented in our model by the adhesion parameter $\alpha$. This probability rises from around $6 \%$ for a neutral mutation occurring at the crypt base to around $40 \%$ for a mutation with a five-fold increase in cell-substrate adhesion.

We found that the probability of domination is affected to a lesser extent by disruption of control of cell proliferation by signalling cues such as Wnt, represented in our model by changes in the proliferation ceiling $y_{\mathrm{thr}}$. In this case we found a counter-intuitive non-monotonic dependence, in which the probability of domination attains a maximum value of $15 \%$ with a proliferation ceiling around $50-60 \%$ of the height of the crypt (assuming no change in adhesion). We hypothesize that this behaviour arises due to an increase in the mean vertical component of the force per unit time on cells at the crypt base, which arises due to elevated levels of proliferation further up the crypt. We suggest that this increase in compression confers a competitive advantage on mutant cells, but that the lateral spread that occurs as cells move up the crypt causes this advantage to diminish in cases where the mutant proliferation ceiling is greater than around $60 \%$ of the crypt height.

We also compared our simulation results with those from by a simple 1D stochastic model of the population dynamics at the base of the crypt. We found that in the case of altered adhesion, there is a straightforward linear relationship between mutant cell-substrate adhesion in our cell-based model and mutant 'fitness' in the simplified model. In contrast, in the case of altered proliferation ceiling, our results indicate the behaviour of cells higher up the crypt affects that of cells at the base when estimating the 
likelihood of mutant domination. In other words, a one-dimensional model of competition at the crypt base is a good simplified model for competition with altered adhesion. But a two-dimensional model that takes account of height up the crypt as well distance around the crypt circumference is required to observe the role of changes to proliferation.

Under the 'top-down' hypothesis carcinogenesis is initiated through a somatic mutation in a cell towards the top of the crypt, which prevents differentiation (Komarova and Wang, 2004). According to our model, even mutants with unlimited proliferative ability, or those with significantly increased cell-cell adhesion, would not colonize the entire crypt unless the mutation occurs near the crypt base. We should note that any mutation that prevents a cell being 'sloughed off' the top of the crypt would change this. The tissue-level behaviour of cell-centre models is not thought to be strongly affected by the spring model used for mechanics (Pathmanathan et al., 2009). In contrast, some cell-vertex or continuum models have observed top-down invasion can occur if adhesive effects are strong (Osborne et al., 2010).

In this study we have considered a highly simplified model of cell proliferation, in which all cells towards the crypt base experience a sufficiently high Wnt stimulus and thus progress through the cell cycle, independently of their size or state of compression. In contrast, some experimental evidence indicates a more complex pattern of cell proliferation, in which cells located at the base of the crypt cycle more slowly than those further up (Wright and Alison, 1984b). One possible explanation for this is contact inhibition of cell division, in which stress due to overcrowding causes a cell to proliferate more slowly, enter quiescence or even undergo apoptosis (Dietrich et al., 2002).

At very high levels of cell-substrate adhesion one might expect that rate of cell movement out of the crypt, under our simple model of mechanical relaxation, to be insufficient to match the rate at which cells are introduced from mitosis. This was not observed at the levels of cell-substrate adhesion considered in this study. If this were the case, rather than reaching a quasi-equilibrium, the number of cells in the crypt may increase exponentially, rapidly reaching physically unrealistic levels. This behaviour has been analysed in a coarse-grained continuum model of an intestinal crypt by Murray et al. (2011). This behaviour would not occur in vivo, and in subsequent models could be avoided through the incorporation of a cell-size dependent cell-cycle model that prevents uncontrolled and excessive cell division at high cell densities. The biological mechanism through which shear stress affects the expression of key components in the Wnt signalling pathway has recently been elucidated (Avvisato et al., 2007; Whitehead et al., 2008); and a recent theoretical model by Basan et al. (2010) proposed a mechanism through which contact inhibition affects the concentrations of $\beta$-catenin and E-cadherin within the cytoplasm. The inclusion of a more detailed description of mechanical feedback on cells' transcriptional and adhesive behaviour therefore offers an exciting direction for future work.

In addition, it is becoming increasingly clear that mutations affecting individual proteins can cause significant alterations in cell function as a result of crosstalk between signalling pathways. This was illustrated by the identification of a positive feedback loop present between the Wnt pathway and the extracellular signal regulated-kinase pathway (Kim et al., 2007). This work highlights the need for more accurate models of the signalling networks underlying cell proliferation and adhesion, as well as an awareness of the extent to which model predictions are robust to the presence of crosstalk between these 
networks.

Another simplification we have made in the present study concerns the geometrical description of the crypt. Following Meineke et al. (2001) and van Leeuwen et al. (2009), we have adopted a cylindrical representation of the crypt. Indeed Snippert et al. (2010) observed that few, if any, cell divisions lead to clonal expansion through the base to the opposite side of the crypt, and our geometry is likely to be a reasonable approximation over much of the crypt height. Nonetheless, the resulting overestimation in the number of cells at the base of the crypt means that the persistence times and probabilities of domination reported here may be overestimates, as discussed by Fletcher et al. (2012). However it is qualitative variation of behaviour with model parameters that is of interest. As discussed in Section 1 , we envisage that the nature of these relationships will be preserved for the more detailed geometries considered recently (Buske et al., 2011; Fletcher et al., 2012).

In order to determine how a proliferative advantage bestowed upon mutant cells within a crypt translates into an increased probability of domination, and how this probability varies with the location of the initial mutation, a large number of model simulations were required. This computational intensity is a problem intrinsic to discrete stochastic models. The problem would be compounded were we to include a more detailed model of how mutations in the Wnt pathway affect proliferation and adhesion at the cellular level, such as that proposed by van Leeuwen et al. (2007). Our simplified geometry and description of cell behaviour has enabled us to perform myriad in silico experiments in order to generate, for the first time, statistical distributions for crypt domination events. One resolution of the problem is to develop coarsegrained models, which replicate the essential features of the original model, but to which established mathematical techniques such as asymptotic and bifurcation analysis may be applied. This remains an area of current research (Fozard et al., 2010; Murray et al., 2010), the key difficulty being how to retain in such coarse-grained models the necessary level of detail and spatial resolution to represent mutations in single cells.

A natural extension is to model the curved, three-dimensional geometry of the crypt in a sufficiently computationally-efficient manner that such statistics may still be obtained, and preliminary work in this direction has been undertaken by Dunn et al. (2012) in modelling the generation of crypt curvature. In this work we have only considered a single crypt. It is becoming clear that inter-crypt interactions may be significant in the establishment of lesions such as colonic adenomas (see Humphries and Wright (2008) and references therein). A natural next step is to consider the processes by which a mutant clonal population, having taken over a single crypt, proceeds to invade and dominate neighbouring crypts. This requires perhaps further mutations to prevent sloughing off at the top of the crypt. A three-dimensional model of the crypt that accounts for interactions with the surrounding stroma, and allows for deformation of the epithelium, would enable investigation of the impact of such events on colorectal cancer. These remain avenues for future research.

\section{Acknowledgements}

The authors gratefully acknowledge financial support from the EPSRC awarded to AGF and GRM as part of the Integrative Biology programme (GR/572023/01). GRM is supported by a GlaxoSmithKline Plc 
Grants and Affiliates award. AGF is funded by the EPSRC and Microsoft Research, Cambridge through grant EP/I017909/1 (www.2020science.net). AGF and PKM acknowledge support from the BBSRC through grant BB/D020190/1. PKM was partially supported by a Royal Society Wolfson Research Merit Award. This publication was based on work supported in part by Award No. KUK-C1-013-04, made by King Abdullah University of Science and Technology (KAUST).

\section{References}

Andersson, E., Sandberg, R., Lendahl, U., 2011. Notch signaling: simplicity in design, versatility in function. Development 138, 3593-3612.

Avvisato, C., Yang, X., Shah, S., Hoxter, B., Li, W., Gaynor, R., Pestell, R., Tozeren, A., Byers, S., 2007. Mechanical force modulates global gene expression and $\beta$-catenin signaling in colon cancer cells. J. Cell Sci. 120, 2672-2682.

Barker, N., van Es, J., Kuipers, J., Kujala, P., van den Born, M., Cozijnsen, M., Haegebarth, A., Korving, J., Begthel, H., Peters, P., et al., 2007. Identification of stem cells in small intestine and colon by marker gene Lgr5. Nature 449, 1003-1007.

Barker, N., Ridgway, R., van Es, J., van de Wetering, M., Begthel, H., van den Born, M., Danenberg, E., Clarke, A., Sansom, O., Clevers, H., 2008. Crypt stem cells as the cells-of-origin of intestinal cancer. Nature 457, 608-611.

Basan, M., Idema, T., Lenz, M., Joanny, J., Risler, T., 2010. A reaction-diffusion model of the cadherin-catenin system: a possible mechanism for contact inhibition and implications for tumorigenesis. Biophys. J. 98, 2770-2779.

Boman, B., Fields, J., Cavanaugh, K., Guetter, A., Runquist, O., 2008. How dysregulated colonic crypt dynamics cause stem cell overpopulation and initiate colon cancer. Cancer Res. 68, 3304-3313.

Bullen, T., Forrest, S., Campbell, F., Dodson, A., Hershman, M., Pritchard, D., Turner, J., Montrose, M., Watson, A., 2006. Characterization of epithelial cell shedding from human small intestine. Lab. Invest. 86, 1052-1063.

Buske, P., Galle, J., Barker, N., Aust, G., Clevers, H., Loeffler, M., Lauffenburger, D., 2011. A comprehensive model of the spatio-temporal stem cell and tissue organisation in the intestinal crypt. PLoS Comp. Biol. 7, 1332-1336.

Dietrich, C., Scherwat, J., Faust, D., Oesch, F., 2002. Subcellular localization of $\beta$-catenin is regulated by cell density. Biochem. Biophys. Res. Commun. 292, 195-199.

Drasdo, D., Loeffler, M., 2001. Individual-based models to growth and folding in one-layered tissues: intestinal crypts and early development. Nonlinear Anal.-Theor. 47, 245-256.

Dunn, S., Fletcher, A., Chapman, S., Gavaghan, D., Osborne, J., 2012. Modelling the role of the basement membrane beneath a growing epithelial monolayer. J. Theor. Biol. 298, 82-91.

Edwards, C., Chapman, S., 2007. Biomechanical modelling of colorectal crypt budding and fission. Bull. Math. Biol. 69, $1927-1942$.

Fletcher, A., Breward, C., Chapman, S., 2012. Mathematical modeling of monoclonal conversion in the colonic crypt. Journal of Theoretical Biology 300, 118-133.

Fozard, J., Byrne, H., Jensen, O., King, J., 2010. Continuum approximations of individual-based models for epithelial monolayers. Math. Med. Biol. 27, 39-74.

Gaspar, C., Fodde, R., 2004. APC dosage effects in tumorigenesis and stem cell differentiation. Int. J. Dev. Biol. 48, $377-386$

Gregorieff, A., Clevers, H., 2005. Wnt signaling in the intestinal epithelium: from endoderm to cancer. Genes Dev. 19, $877-890$

Harris, T., Peifer, M., 2005. Decisions, decisions: $\beta$-catenin chooses between adhesion and transcription. Trends Cell Biol. 15, 234-237.

Humphries, A., Wright, N., 2008. Colonic crypt organization and tumorigenesis. Nat. Rev. Cancer 8, 415-424.

Ilyas, M., 2005. Wnt signalling and the mechanistic basis of tumour development. J. Pathol. 205, 130-144.

Johnston, M., Edwards, C., Bodmer, W., Maini, P., Chapman, S., 2007. Mathematical modeling of cell population dynamics in the colonic crypt and in colorectal cancer. Proc. Nat. Acad. Sci. U. S. A. 104, 4008-4013.

Kaur, P., Potten, C., 1986. Effects of puromycin, cycloheximide and noradrenaline on cell migration within the crypts and on the villi of the small intestine. A model to explain cell movement in both regions. Cell Prolif. 19, 611-. 
Kim, D., Rath, O., Kolch, W., Cho, K., 2007. A hidden oncogenic positive feedback loop caused by crosstalk between Wnt and ERK pathways. Oncogene 26, 4571-4579.

Komarova, N., 2007. Loss-and gain-of-function mutations in cancer: mass-action, spatial and hierarchical models. J. Stat. Phys. 128, 413-446.

Komarova, N., Wang, L., 2004. Initiation of colorectal cancer. Cell Cycle 3, 1558-1565.

Loeffler, M., Birke, A., Winton, D., Potten, C., 1993. Somatic mutation, monoclonality and stochastic models of stem cell organization in the intestinal crypt. J. Theor. Biol. 160, 471-471.

Lopez-Garcia, C., Klein, A., Simons, B., Winton, D., 2010. Intestinal stem cell replacement follows a pattern of neutral drift. Science 330, 822-825.

Meineke, F., Potten, C., Loeffler, M., 2001. Cell migration and organization in the intestinal crypt using a lattice-free model. Cell Prolif. 34, 253-266

Mirams, G., 2008. Subcellular phenomena in colorectal cancer. Ph.D. thesis. University of Nottingham.

Mirams, G., Byrne, H., King, J., 2010. A multiple timescale analysis of a mathematical model of the Wnt/ $\beta$-catenin signalling pathway. J. Math. Biol. 60, 131-160.

Morin, P., Sparks, A., Korinek, V., Barker, N., Clevers, H., Vogelstein, B., Kinzler, K., 1997. Activation of $\beta$-catenin-Tcf signaling in colon cancer by mutations in $\beta$-catenin or APC. Science 275, 1787- 1790.

Murray, P., Kang, J., Mirams, G., Shin, S., Byrne, H., Maini, P., Cho, K., 2010. Modelling spatially regulated $\beta$-catenin dynamics and invasion in intestinal crypts. Biophys. J. 99, 716-725.

Murray, P., Walter, A., Fletcher, A., Edwards, C., Tindall, M., Maini, P., 2011. Comparing a discrete and continuum model of the intestinal crypt. Phys. Biol. 8, 026011.

Nelson, W., Nusse, R., 2004. Convergence of Wnt, $\beta$-catenin, and cadherin pathways. Science 303, $1483-1487$.

Nowak, M., Michor, F., Iwasa, Y., 2003. The linear process of somatic evolution. Proc. Nat.Acad. Sci. USA 100, 1496614969.

Okamoto, R., Watanabe, M., 2004. Molecular and clinical basis for the regeneration of human gastrointestinal epithelia. J.Gastroenterol. 39, 1-6.

Osborne, J., Walter, A., Kershaw, S., Mirams, G., Fletcher, A., Pathmanathan, P., Gavaghan, D., Jensen, O., Maini, P., Byrne, H., 2010. A hybrid approach to multi-scale modelling of cancer. Phil. Trans. R. Soc. A 368, 5013-5028.

Pathmanathan, P., Cooper, J., Fletcher, A., Mirams, G., Murray, P., Osborne, J., Pitt-Francis, J., Walter, A., Chapman, S.J., 2009. A computational study of discrete mechanical tissue models. Physical Biology 6, 036001.

Perez-Moreno, M., Jamora, C., Fuchs, E., 2003. Sticky business orchestrating cellular signals at adherens junctions. Cell $112,535-548$.

Pinto, D., Gregorieff, A., Begthel, H., Clevers, H., 2003. Canonical Wnt signals are essential for homeostasis of the intestinal epithelium. Genes Dev. 17, 1709-1713.

Pitt-Francis, J., Pathmanathan, P., Bernabeu, M., Bordas, R., Cooper, J., Fletcher, A., Mirams, G., Murray, P., Osborne, J., Walter, A., Chapman, J., Garny, A., van Leeuwen, I., Maini, P., Rodriguez, B., Waters, S., Whiteley, J., Byrne, H., Gavaghan, D., 2009. Chaste: a test-driven approach to software development for biological modelling. Comp. Phys. Comm. 180, 2452-2471.

Polakis, P., 2007. The many ways of Wnt in cancer. Curr. Opin. Genet. Dev. 17, 45-51.

Potten, C., Booth, C., Hargreaves, D., 2003. The small intestine as a model for evaluating adult tissue stem cell drug targets. Cell Prolif. 36, 115-129.

Powell, S., Zilz, N., Beazer-Barclay, Y., Bryan, T., Hamilton, S., Thibodeau, S., Vogelstein, B., Kinzler, K., 1992. APC mutations occur early during colorectal tumorigenesis. Nature 359, $235-237$.

Ross, M., Kaye, G., Pawlina, W., 2003. Histology: a text and atlas: with cell and molecular biology. Lippincott Williams \& Wilkins.

Sansom, O., Meniel, V., Muncan, V., Phesse, T., Wilkins, J., Reed, K., Vass, J., Athineos, D., Clevers, H., Clarke, A., 2007. Myc deletion rescues Apc deficiency in the small intestine. Nature 446, 676-679.

Sansom, O., Reed, K., Hayes, A., Ireland, H., Brinkmann, H., Newton, I., Batlle, E., Simon-Assmann, P., Clevers, H., Nathke, I., Clarke, A., Winton, D., 2004. Loss of Apc in vivo immediately perturbs Wnt signaling, differentiation, and migration. Genes Dev. 18, 1385-1390.

Shih, I., Wang, T., Traverso, G., Romans, K., Hamilton, S., Ben-Sasson, S., Kinzler, K., Vogelstein, B., 2001. Top-down morphogenesis of colorectal tumors. Proc. Nat. Acad. Sci. U. S. A. 98, 2640-2645.

Snippert, H., van der Flier, L., Sato, T., van Es, J., van den Born, M., Kroon-Veenboer, C., Barker, N., Klein, A., van 
Rheenen, J., Simons, B., Clevers, H., 2010. Intestinal crypt homeostasis results from neutral competition between symmetrically dividing Lgr5 stem cells. Cell 143, 134-144.

Sparks, A., Morin, P., Vogelstein, B., Kinzler, K., 1998. Mutational analysis of the APC/ $\beta$-catenin/Tcf pathway in colorectal cancer. Cancer Res. 58, 1130-1134.

Sunter, J., Appleton, D., Dé Rodriguez, M., Wright, N., Watson, A., 1979. A comparison of cell proliferation at different sites within the large bowel of the mouse. J. Anat. 129, 833-42.

van de Wetering, M., Sancho, E., Verweij, C., de Lau, W., Oving, I., Hurlstone, A., van der Horn, K., Batlle, E., Coudreuse, D., Haramis, A., Tjon-Pon-Fong, M., Moerer, P., van den Born, M., Soete, G., Pals, S., Eilers, M., Medema, R., Clevers, H., 2002. The $\beta$-catenin/TCF-4 complex imposes a crypt progenitor phenotype on colorectal cancer cells. Cell 111, $241-250$.

van der Flier, L., Clevers, H., 2009. Stem cells, self-renewal, and differentiation in the intestinal epithelium. Annu. Rev. Physiol. 71, 241-260.

van Leeuwen, I., Byrne, H., Jensen, O., King, J., 2007. Elucidating the interactions between the adhesive and transcriptional functions of $\beta$-catenin in normal and cancerous cells. J. Theor. Biol. 247, 77-102.

van Leeuwen, I., Mirams, G., Walter, A., Fletcher, A., Murray, P., Osborne, J., Varma, S., Young, S., Cooper, J., Doyle,

B., Pitt-Francis, J., Momtahan, L., Pathmanathan, P., Whiteley, J., Chapman, S.J., Jensen, O., King, J., Maini, P.,

Waters, S., Gavaghan, D., Byrne, H., 2009. An integrative computational model for intestinal tissue renewal. Cell Prolif. $42,617-636$.

Whitehead, J., Vignjevic, D., Fütterer, C., Beaurepaire, E., Robine, S., Farge, E., 2008. Mechanical factors activate $\beta$-catenin-dependent oncogene expression in apc1638n/+ mouse colon. HFSP J. 2, 286-294.

Wright, N., Alison, M., 1984a. The biology of epithelial cell populations: volume 1. Clarendon Press, Oxford.

Wright, N., Alison, M., 1984b. The biology of epithelial cell populations: volume 2. Clarendon Press, Oxford. 



\section{RECENT REPORTS}

06/12 Effects of intrinsic stochasticity on delayed reaction-diffusion patterning systems

Woolley

Baker

Gaffney

Maini

Seirin-Lee

07/12 Axial Dispersion via Shear-enhanced Diffusion in Colloidal Suspensions

Griffiths

Stone

08/12 Qualitative Analysis of an Integro-Differential Equation Model of Jaina Periodic Chemotherapy Byrne

09/12 Modeling Stem/Progenitor Cell-Induced Neovascularization and Jain Oxygenation

Moldovan

Byrne

10/12 Allee Effects May Slow the Spread of Parasites in a Coastal Marine Ecosystem

Krkošek

Connors

Lewis

Poulin

11/12 Parasite spill-back from domestic hosts may induce an Allee effect in wildlife hosts

Krkošek

Ashander

Lewis

12/12 Modelling temperature-dependent larval development and subse-

Wheeler quent demographic Allee effects in adult populations of the alpine butterfly Parnassius smintheus

Bampfylde

Lewis

13/12 Putting "space" back into spatial ecology Fortin

Peres-Neto

Lewis

14/12 Wildlife disease elimination and density dependence Potapova

Merrill

Lewis

15/12 Spreading Speed, Traveling Waves, and Minimal Domain Size in

Lewis

Impulsive Reaction-diffusion Models

$\mathrm{Li}$

16/12 MCMC methods for functions modifying old algorithms to make them faster

Cotter

Roberts

Stuart

White

17/12 Weyl Geometry and the Nonlinear Mechanics of Distributed Point Defects

Yavari

Goriely

18/12 A note on oblique water entry

Moore

Howison

Ockendon

Oliver

19/12 Calculus on surfaces with general closest point functions

März

Macdonald 
20/12 Multiple equilibria in a simple elastocapillary system

Taroni

Vella

21/12 Multiphase modelling of vascular tumour growth in two spatial

Hubbard dimensions

Byrne

22/12 Chebfun and Numerical Quadrature

Hale

Trefethen

23/12 Moment-based formulation of NavierMaxwell slip boundary con-

Reis ditions for lattice Boltzmann simulations of rarefied flows in microchannels

24/12 Correspondence between one- and two-equation models for solute transport in two-region heterogeneous porous media

Dellar

Davit

Wood

Debenest

Quintard

25/12 Rolie-Poly fluid flowing through constrictions: Two distinct instabilities

Reis

Wilson

26/12 Age related changes in speed and mechanism of adult skeletal muscle stem cell migration

Collins-Hooper

Woolley

Dyson

Patel1

Potter

Baker

Gaffney

Maini

Dash

Patel

27/12 The interplay between tissue growth and scaffold degradation in

ODea engineered tissue constructs

Osborne

El Haj

Byrne

Waters

28/12 Non-linear effects on Turing patterns: time oscillations and chaos.

Aragon

Barrio

Woolley

Baker

Maini

29/12 Colorectal Cancer Through Simulation and Experiment

Kershaw

Byrne

Gavaghan

Osborne

Copies of these, and any other OCCAM reports can be obtained 
from:

Oxford Centre for Collaborative Applied Mathematics Mathematical Institute

24 - 29 St Giles'

Oxford

OX1 3LB

England

www.maths.ox.ac.uk/occam 